\title{
Conducta ingestiva y preferencia bovina por el ensilaje de Pennisetum y pulpa de café ${ }^{1}$
}

\author{
Intake behavior and preference of cattle by Pennisetum silage mixed \\ with coffee pulpa
}

\author{
René Pinto-Ruiz ${ }^{2}$, Francisco Guevara-Hernández $z^{2}$, José Alfredo Medina ${ }^{2}$, David Hernández-Sánchez ${ }^{3}$, \\ Alejandro Ley-de Coss ${ }^{4}$, Enrique Guerra-Medina ${ }^{4}$
}

\section{RESUMEN}

El objetivo de este trabajo fue evaluar la sustitución de $P$. purpureum var OM-22 por pulpa de café $(25,50,75$ y $100 \%$ ) en la fabricación de ensilajes. El trabajo se realizó de enero a marzo de 2015 en la región central de Chiapas, México. En el animal se midió: velocidad de bocados, velocidad de consumo, tamaño de bocado y el índice de preferencia; en el ensilaje las variables organolépticas: olor, color, humedad y textura, y la composición química a través del análisis de proteína cruda, fibra detergente neutro y ácido, materia orgánica, cenizas, taninos condensables, glucósidos cianogénicos, saponinas y cafeína, así como $\mathrm{pH}$. Los datos de calidad y conducta ingestiva fueron sometidos a un análisis de varianza mediante el procedimiento GLM del paquete estadístico SAS y las medias fueron comparadas utilizando la prueba de Tukey $(\mathrm{p}<0,05)$. Los resultados indicaron que es factible la incorporación de pulpa de café entre un 25 y $50 \%$, ya que el índice de preferencia $(0,40$ y 0,35$)$ y la velocidad de consumo $(10,20$ y 8,81$)$ fueron mayores $(p<0,05)$, en comparación con los otros porcentajes de sustitución, producto de las características organolépticas del ensilaje que, en general, fueron recomendables, así como por la mejora en la calidad química en términos de proteína cruda y ausencia de metabolitos secundarios.

Palabras claves: variables organolépticas, calidad nutricional, velocidad de consumo.

\begin{abstract}
The aim of this study was to evaluate the replacement of $P$. purpureum var OM-22 for coffee pulp $(25,50,75$ and $100 \%)$ in the production of silage. The work was conducted from January to March 2015 in the central region of Chiapas, Mexico. Bites rate, consumption rate, bite size and preference index was measured on the animals; organoleptic variables: odor, color, moisture and texture and chemical composition through the analysis of crude protein, neutral detergent fiber and acid, organic matter, ash, condensable tannins, cyanogenic glycosides, saponins and cafeine, also $\mathrm{pH}$ was evaluated in the silage. Data from quality and ingestive behavior were subjected to analysis of variance using the GLM procedure of SAS statistical package and the means were compared using the Tukey test $(\mathrm{p}<0.05)$. The results indicated that the incorporation of coffee pulp by 25 to $50 \%$ is feasible since the preference index $(0.40$ y 0.35$)$ and consumption rate $(10.20$ y 8.81$)$ was higher $(\mathrm{p}<0.05)$, in comparison with other percentages that resulted from the organoleptic characteristics of silage as well as the improvement in chemical quality in terms of crude protein and absence of secondary metabolites in general.
\end{abstract}

Keywords: organoleptic variables, nutritional quality, consumption rate.

\footnotetext{
Recibido: 9 de febrero, 2016. Aceptado: 9 de mayo, 2016. Este trabajo formó parte del proyecto de investigación "Uso de subproductos de café derivados de la producción del café Coffea arabica (pulpa, mucilago y cascarilla)" financiado por Sistema Institucional de Investigación de la Universidad Autónoma de Chiapas, México.

2 Universidad Autónoma de Chiapas, Facultad de Ciencias Agronómicas. Carretera Ocozocoautla-Villaflores, km 80. Villaflores, Chiapas, México.CP 30460.pinto_ruiz@yahoo.com.mx (autor para correspondencia), fragueher@prodigy.net.mx, jmedina_2157@hotmail.com

3 Colegio de Postgraduados, Campus Montecillo, Carretera México-Texcoco km 36.5 Montecillo, Texcoco, Estado de México, México. CP 56230.sanchezd@colpos.mx

4 Universidad Autónoma de Chiapas, Facultad de Ciencias Agrícolas. Entronque Carretera Costera y Estación Huehuetán. Huehuetan, Chiapas, México. CP 30660. aleycoss@gmail.com, enrique.guerra@cucsur.udg.mx
} 


\section{INTRODUCCIÓN}

Uno de los problemas más limitantes que se presenta en la ganadería bovina tropical es la baja producción de las pasturas, específicamente durante la época seca, recurriéndose, por ello, a la utilización de diversas estrategias de suplementación. Una alternativa comúnmente empleada para contrarrestar este problema, ha sido la conservación de materiales forrajeros a través de ensilajes, con base en diversas especies de gramíneas, follajes arbóreos y otras fuentes potencialmente forrajeras, tales como algunos subproductos (Solomon, 1991; Pinto et al., 2016). El reto es ofrecer a los animales alimento de buena calidad aprovechando los recursos locales.

Chiapas, en el sureste mexicano, cuenta con más de 245000 ha cultivadas de café, es líder mundial en la producción de café orgánico y un sólido participante en el comercio justo, ocupa el primer lugar de producción a nivel nacional (SAGARPA, 2011). Sin embargo, en la producción y procesamiento del café se generan grandes cantidades de subproductos (se estima que alrededor de 152000 toneladas de pulpa de café son producidas anualmente en la región), que no se utilizan y que llegan a ser contaminantes del agua y suelo.

Ante ello, se han generado diversas alternativas de uso de dicho subproducto para evitar, con ello, procesos de contaminación. En ese sentido, la pulpa de café ha sido utilizada en el mundo para la producción de biogás, obtención de abono orgánico, producción de hongos comestibles, obtención de alcohol, vinos, obtención de carbón activado y en la alimentación animal (Salmones et al., 2005; Nurfeta, 2010; Kassa et al., 2011; Pedraza et al., 2012; Corro et al., 2013; Menezes et al., 2014).

En los sistemas de alimentación animal, principalmente en rumiantes, el uso de la pulpa de café ha sido mayormente a través de procesos de ensilaje (Moreau et al., 2003; Rathinavelu y Graziosi, 2005). Sobre el tema, se han realizado trabajos muy completos sobre el proceso de ensilaje y sus costos (Murillo, 1978); sobre su composición (Noriega et al., 2009), el uso de aditivos (Molina et al., 1990; Ferrer et al., 1995) y en revisiones realizadas por Noriega et al. (2008), Pinto et al. (2010) y Munguía (2015), se reportaron sus bondades y restricciones de uso en diferentes especies animales. Pero los trabajos han sido orientados al ensilaje de la pulpa sola, sin considerar al componente animal en su preparación.

$\mathrm{Al}$ respecto, se reconoce que para la introducción de nuevas tecnologías en los sistemas de alimentación animal, se requieren conocimientos acerca de la preferencia animal, ya que se ha documentado que la preferencia y, por tanto, el consumo de cualquier alimento o suplemento determina, en primer instancia, el nivel de producción animal y establece el uso potencial que puede obtenerse de este, por lo que reviste gran importancia su evaluación (Preston y Leng, 1990), para lo cual es necesario utilizar al animal como seleccionador final del alimento.

El objetivo de este trabajo fue evaluar la sustitución de Pennisetum purpureum var OM-22 por pulpa de café $(0,25,50,100)$ en la fabricación de ensilajes.

\section{MATERIALES Y MÉTODOS}

\section{Características del área de estudio}

La evaluación se realizó de enero a marzo de 2015 en la Estación Experimental de la Facultad de Ciencias Agronómicas de la Universidad Autónoma de Chiapas, en el municipio de Villaflores, perteneciente al estado de Chiapas, situado entre los $16^{\circ} 13^{\prime} 15$ " N y $93^{\circ} 16^{\prime} 07^{\prime \prime} \mathrm{O}$, a una altitud de $610 \mathrm{~m}$. El clima es cálido subhúmedo, la temperatura media anual es de $24,3{ }^{\circ} \mathrm{C}$ y la precipitación media anual de $1,209 \mathrm{~mm}$, que ocurre principalmente en el verano (García, 1989).

\section{Tratamientos}

Los tratamientos consistieron en la sustitución de Pennisetum purpureum var OM-22 por cuatro niveles de pulpa de café $(25 \%, 50 \%, 75 \%$ y $100 \%)$.

\section{Obtención de la pulpa de café}

La pulpa de café se obtuvo directamente del proceso de beneficio húmedo llevado a cabo en las comunidades indígenas cafetaleras aledañas al municipio de Jaltenango, localizado en la región Frailesca (Chiapas, México), en la cosecha de 2014 (diciembre), la cual fue almacenada en bolsas de plástico para su uso inmediato. 


\section{Elaboración de los microsilos}

El ensilado se realizó en bolsas plásticas de polietileno de color negro con una capacidad de 35 $\mathrm{kg}$, de las que se ha demostrado su efectividad en el proceso (Ashbell et al., 2001). Las proporciones de pasto y pulpa correspondientes a cada tratamiento fueron introducidas dentro de las bolsas siguiendo la metodología propuesta por Ojeda et al. (1991). Una vez terminado el proceso, las bolsas fueron almacenadas con cuidado en un sitio a temperatura ambiente, fuera del alcance de roedores y de otras fuentes de peligro. Los microsilos fueron abiertos a los noventa días posteriores a su elaboración, se utilizaron en la prueba de preferencia animal y en la realización de las pruebas organolépticas y químicas.

El pasto Pennisetum purpureum var OM-22 utlizado fue establecido en una parcela de corte y acarreo, sin riego ni fertilización. El material se cosechó a una edad de rebrote de tres meses y a una altura de $2,5 \mathrm{~m}$.

\section{Variables evaluadas}

A través de un panel de observadores, previamente entrenados y con experiencia, se realizaron pruebas organolépticas de los distintos tratamientos (olor, color, humedad y textura) de acuerdo con una escala referencial (Ojeda et al., 1991). Esta evaluación se basó en la apreciación subjetiva de la calidad del ensilaje a través de los sentidos. Las calificaciones se distribuyeron en tres rangos de calificación.

A cada uno de los tratamientos se les determinó el $\mathrm{pH}$ a través de un potenciómetro Orion Research SA 210; para conocer la composición química se analizaron diez muestras compuestas (repeticiones), secadas en estufa de aire forzado a $60{ }^{\circ} \mathrm{C}$ y molidas individualmente en molino de motor tipo Wiley. Se obtuvieron diez submuestras de aproximadamente 300 $\mathrm{g}$, con un tamaño de molido aproximado de $1 \mathrm{~mm}$. A estas se les determinó el contenido de proteína cruda (PC), materia orgánica (MO) y cenizas (CE) de acuerdo con los procedimientos de la AOAC (1990); la fracción de fibra detergente neutro y fibra detergente ácido (FDN y FDA), mediante la técnica de Van Soest et al. (1991); los taninos condensables (TC) con la metodología descrita por Terril et al. (1992); glucósidos cianogénicos (GC) y saponinas (SA) con las técnicas descritas por
Arrázola (2002); y cafeína, cuantificada mediante el proceso recomendado por Morris (1973).

La preferencia y conducta ingestiva se evaluó mediante una prueba de cafetería, basada en la observación directa del animal (Kaitho, 1997), utilizando para ello cinco corrales individuales, en los que, en cada uno, fue confinado un torete cebú-suizo, encaste típico de la región, con un peso promedio de $235 \pm 12,3$ $\mathrm{kg}$ y una edad aproximada de dos años, con rumen totalmente desarrollado. Previamente, cada animal fue desparasitado subcutáneamente (Doramectina 1\%, 1 $\mathrm{ml}$ por cada $50 \mathrm{~kg}$ de peso); además, se les aplicó instramuscularmente un complejo vitamínico ADE (4 $\mathrm{ml}$ por animal). Los animales permanecieron en pastoreo rotacional de praderas de estrella africana (Cynodon plectostachyus) todo el día, excepto por la tarde, que fueron confinados en los corrales (14:00 a18:00 h) con comederos de madera con cinco compartimientos. A los animales confinados en su corral, les fueron ofrecidos los cinco tratamientos de manera simultánea, colocados aleatoriamente en los compartimentos del comedero durante cada día de evaluación, de tal forma que ocuparan todos los lugares posibles dentro del comedero, con la finalidad de bloquear el hábito reflejo de los animales a la posición, la distancia del microsilo y el primer encuentro con el suplemento. Los animales fueron adaptados a los ensilajes durante un período de dieciocho días. La conducta ingestiva fue caracterizada a través de las variables: velocidad de bocados (bocados/ $\mathrm{min}$ ), velocidad de consumo (g MS/min) y tamaño de bocado (g MS/bocado), se utilizó para ello la técnica de muestreo focal, basada en la observación continua y directa del animal (Altmann, 1974), y en los procedimientos sugeridos para ello (Sosa et al., 2004). El índice de preferencia (IP) se obtuvo al considerar el número de veces consumiendo " $n$ " tratamiento entre el número total de veces que se consumieron todos los tratamientos (Ortega y Rivas, 1998).

\section{Diseño experimental y análisis de datos}

Los datos fueron sometidos a análisis de varianza simple mediante el procedimiento GLM del paquete estadístico SAS (SAS, 1994). Para analizar las variables de calidad, se utilizó un diseño experimental completamente al azar con cinco tratamientos (tipo de ensilaje) y diez repeticiones por tratamiento. Para la prueba de conducta y preferencia, el experimento 
tuvo un periodo de colecta de datos de cinco días y se utilizó un diseño experimental cuadrado latino (5x5). En ambos casos, las medias fueron evaluadas utilizando la prueba de Tukey $(\mathrm{p}<0,05)$.

\section{RESULTADOS Y DISCUSIÓN}

La composición, previa (\%) al ensilado, del $P$. purpureum (MS:32,33, PC:4,22, MO:92,47, Ce:7,52, FDN;65,11, FDA:30,12) y de la pulpa de café (MS:24,51, PC:12,99, MO:89,70, Ce:10,30, FDN:44,32, FDA:38,04, TC:2,12, GC:neg, SA:neg, Cafeína:0,73) indicó que el proceso de ensilado produjo cambios en el material original, sobre todo con relación con los metabolitos secundarios, pero sin afectar significativamente el valor nutricional.

En el Cuadro 1 se presentan los resultados de la evaluación de las distintas variables organolépticas en los distintos tipos de ensilajes. Los datos fueron expresados en frecuencias absolutas.

Junto con un análisis químico, factores como el olor, color y el aspecto general proporcionan un buen indicador del valor nutritivo global esperado de materiales de ensilaje (ARD, 2004). En ese sentido, en cuanto a olor, los tratamientos con $0 \%$ y $25 \%$ de inclusión de pulpa de café fueron los mejores calificados, pues presentaron un olor agradable (fruta fermentada), a diferencia del resto de los tratamientos que presentaron olores menos agradables y ligeros a vinagre. Esto indica que al aumentar el porcentaje de inclusión, el olor tiende a ser menos agradable, ya que la pulpa de café expide un olor característico por efecto de su fermentación. La ventaja del buen olor está relacionada con la preferencia y mayor consumo por parte del animal.

Se reconoce que la apariencia y el color del ensilaje de pulpa de café son característicos del proceso de ensilado, después de haber desarrollado una fermentación anaeróbica adecuada (Solomon, 1991). Al respecto, los tratamientos con $0 \%$ y $25 \%$ de inclusión de pulpa de café presentaron un color más parecido al original (antes de ensilar), contrario al resto de los tratamientos, los cuales presentaron un color más oscuro y diferente al original, lo cual se debió a que la pulpa de café tiende a cambiar su color original a uno más oscuro por efecto de la oxidación. Se ha reportado que existe un cambio en el color
Cuadro 1. Frecuencias de calificaciones en la evaluación organoléptica de los ensilajes elaborados con niveles crecientes de pulpa de café (Coffea arabica L.) en sustitución de Pennisetum purpureum var OM-22 en Villaflores, Chiapas, México durante 2015.

Table 1. Frequencies of the sensory evaluation scores of silage made with increasing levels of pulp of coffee (Coffea arabica L.) in place of Pennisetum purpureum var OM-22 in Villaflores, Chiapas, México during 2015.

\begin{tabular}{lcccc}
\hline Variables & $\begin{array}{c}\text { Tratamientos } \\
\text { (\% pulpa) }\end{array}$ & Bueno & Regular & Malo \\
\hline Olor & 0 & 100 & & \\
& 25 & 66,7 & 33,3 & \\
& 50 & & 77,8 & 22,2 \\
& 75 & & 66,7 & 33,3 \\
& 100 & 22,2 & 66,7 & 11,1 \\
\hline Color & 0 & 100 & & \\
& 25 & 100 & & \\
& 50 & 33,3 & 55,6 & 11,1 \\
& 75 & 11,1 & 77,8 & 11,1 \\
& 100 & 11,1 & 55,6 & 33,3 \\
\hline Humedad & 0 & 33,3 & 44,4 & 22,2 \\
& 25 & 22,2 & 55,6 & 22,2 \\
& 50 & & 66,7 & 33,3 \\
& 75 & & 66,7 & 33,3 \\
& 100 & & 44,4 & 55,6 \\
\hline Textura & 0 & 100 & & \\
& 25 & 77,8 & 22,2 & \\
& 50 & 55,6 & 44,4 & \\
& 75 & 55,6 & 44,4 & \\
& 100 & 55,6 & 33,3 & 11,1 \\
\hline
\end{tabular}

de la pulpa de café de color marrón a negro cuando los ensilajes se exponen al aire, lo cual se atribuye a reacciones enzimáticas causadas por la oxidación de los polifenoles o quinonas, las que, a su vez, se combinan con aminoácidos libres y proteínas para dar complejos de coloración oscura (Solomon, 1991). Por su lado, los tratamientos con 50, 75 y $100 \%$ de pulpa fueron mayormente calificados como regulares (Cuadro 1). 
Respecto a humedad, los tratamientos $0,25,50$ y $75 \%$ fueron calificados mayormente como regulares en su contenido de agua, sin embargo, el tratamiento con $100 \%$ pulpa presentó mucha humedad. El alto contenido de humedad de los silos influye en la calidad fermentativa y nutritiva final, por lo que podría afectar el rechazo por parte de los animales. Finalmente, en cuanto a textura, todos los tratamientos fueron calificados como buenos y regulares, ya que, tanto la pulpa como el forraje conservaron todos sus contornos bien definidos, es decir, presentaron sus características originales.

En los Cuadros 2 y 3 se observan los resultados del análisis químico practicado a los diferentes ensilajes. Se conoce que el proceso de beneficiado y la variedad del café pueden influir en la cantidad de MS del ensilado. En este trabajo los valores de MS del ensilaje de café se encontraron dentro de los rangos reportados por Bautista et al. (2005) y Munguía (2015). A pesar del bajo valor promedio de MS de los tratamientos conteniendo pulpa de café $(21,70 \%)$, se supera el mínimo requerido $(20,00 \%)$ para asegurar una buena calidad en la fermentación (Munguía, 2015). Por otro lado, los valores de MS del ensilaje $(32,8 \%)$ se encontraron dentro del rango considerado como óptimo (30-35\%) según Ojeda et al. (1991), y muy similar al reportado por Cárdenas et al. (2003). Estos valores desempeñan un papel muy importante en la calidad fermentativa y nutritiva final del ensilaje, al controlar el crecimiento de Clostridium, disminuir la actividad fermentativa y la producción de efluentes e incrementar el consumo (Ojeda et al., 1991).

La importancia del $\mathrm{pH}$ radica en su vinculación con los procesos degradativos que se producen en la conservación. Los valores de $\mathrm{pH}$ obtenidos en este trabajo (Cuadro 2) indicaron que ensilar pulpa de café proporciona condiciones ambientales favorables, pues se ha indicado que valores de 4,5 son indicadores de una fermentación anaeróbica adecuada, y cuando se alcanzan valores de 4,2 se logra estabilidad fermentativa (Ojeda et al., 1991). Los valores encontrados en este trabajo superaron ligeramente los reportados por Munguía (2015) $(3,90)$ y Villalba et al. $(2011)(3,8)$ al ensilar únicamente pulpa de café, y fueron muy similares a los obtenidos por Yonatan et al. (2011) al ensilar pulpa de café con pasto.

Con respecto al porcentaje de PC, se evidenció la factibilidad de ensilar hasta con un $100 \%$ de pulpa, pues los valores de este nutriente aumentaron conforme el porcentaje de inclusión de pulpa aumentó en el ensilaje $(\mathrm{p}<0,05 \%)$. De igual manera, respecto al contenido de materia orgánica, el análisis reveló el incremento de los valores $(\mathrm{p}<0,05)$ al aumentar el porcentaje de inclusión de pulpa de café, caso contrario ocurrió con los valores de FDN y FDA, los cuales tendieron a disminuir significativamente $(\mathrm{p}<0,05)$ conforme el porcentaje de inclusión de pulpa de café aumentó. Por otra parte, para taninos condensados (TC), únicamente el tratamiento con $100 \%$ de pulpa reportó valores $(\mathrm{p}<0,05)$ distintos de 0 . No se encontró, en la prueba cualitativa, presencia de glucósidos cianogénicos ni saponinas en ninguno de los tratamientos.

Los valores proteínicos de los ensilajes, en los cuales se incorporó la pulpa de café $(25,50,75$ y $100 \%$ ), fueron mayores respecto al ensilaje de $P$. purpureum solo, lo cual respalda la información referente a que el uso de este subproducto mejora la calidad de esta práctica de conservación de forrajes (Murillo, 1978). Al respecto, González (1990) ha clasificado a los forrajes y otros alimentos para animales como de regular calidad cuando contienen valores entre 7 y $9 \%$ de proteína, y de buena calidad con valores comprendidos entre 9 y $11 \%$, lo que significa que a partir del $50 \%$ de inclusión de pulpa de café los ensilajes presentaron un contenido de proteína cruda que los ubica como alimentos de un valor nutricional alto. Valores de entre 10,7 y $11,5 \%$ de proteína cruda, en ensilajes con gramíneas y pulpa de café $(10,20,30$ y $100 \%$ de sustitución) fueron obtenidos por Yonatan et al. (2011), los cuales fueron similares a los obtenidos en este experimento $(10,13 \%$ en promedio).

Los mayores valores de cenizas encontradas en el tratamiento sin pulpa $(0 \%)$, podrían estar asociados al aporte de fibra por parte de los tallos de la gramínea, ya que los pastos poseen un mayor contenido de componentes de la pared celular que la pulpa de café (Yonatan et al., 2011). Los valores de cenizas y fibras fueron similares a los reportados en el trabajo de Yonatan et al. (2011).

Muchos trabajos han establecido que la pulpa de café posee metabolitos secundarios que limitan su uso en la alimentación animal (Clifford, 1985; Ramírez et al., 2004; Arellano, 2009; Noriega et al., 2009; Torres et al., 2011), identificando que dentro de tales factores los más importantes son cafeína, taninos y polifenoles. Se considera que los taninos junto 
Cuadro 2. Composición química (\%) y pH de ensilajes elaborados con diferentes porcentajes de pulpa de café (Coffea arabica L.) en sustitución de Pennisetum purpureum var OM-22 en Villaflores, Chiapas, México durante 2015.

Table 2. Chemical composition (\%) and $\mathrm{pH}$ of silage made with different percentages of pulp coffee (Coffea arabica L.) in place of Pennisetum purpureum var OM-22 in Villaflores, Chiapas, México during 2015.

\begin{tabular}{lccccccc}
\hline $\begin{array}{l}\text { Tratamientos } \\
\text { (\% pulpa) }\end{array}$ & MS & pH & PC & MO & CE & FDN & FDA \\
\hline 0 & $32,86^{\mathrm{a}^{*}}$ & 5,03 & $\begin{array}{c}4,17^{\mathrm{c}} \\
( \pm 5,07)\end{array}$ & $\begin{array}{c}86,97^{\mathrm{d}} \\
( \pm 0,12)\end{array}$ & $\begin{array}{c}13,03^{\mathrm{a}} \\
( \pm 0,12)\end{array}$ & $\begin{array}{c}61,01^{\mathrm{a}} \\
( \pm 1,54)\end{array}$ & $\begin{array}{c}48,81^{\mathrm{a}} \\
( \pm 1,10)\end{array}$ \\
\hline 25 & $20,78^{\mathrm{b}}$ & 4,51 & $\begin{array}{c}7,26^{\mathrm{b}} \\
( \pm 2,45)\end{array}$ & $\begin{array}{c}90,24^{\mathrm{c}} \\
( \pm 0,05)\end{array}$ & $\begin{array}{c}9,76^{\mathrm{b}} \\
( \pm 0,05)\end{array}$ & $\begin{array}{c}55,80^{\mathrm{b}} \\
( \pm 2,69)\end{array}$ & $\begin{array}{c}45,18^{\mathrm{a}} \\
( \pm 2,65)\end{array}$ \\
\hline 50 & $(1,10)$ & & $10,19^{\mathrm{ab}}$ & $90,38^{\mathrm{c}}$ & $9,62^{\mathrm{b}}$ & $49,00^{\mathrm{c}}$ & $41,79^{\mathrm{ab}}$ \\
& $21,49^{\mathrm{b}}$ & 4,49 & $( \pm 0,02)$ & $( \pm 0,11)$ & $( \pm 0,11)$ & $( \pm 0,17)$ & $( \pm 0,45)$ \\
\hline 75 & $(0,06)$ & & $11,16^{\mathrm{a}}$ & $91,46^{\mathrm{b}}$ & $8,54^{\mathrm{c}}$ & $45,85^{\mathrm{c}}$ & $39,59^{\mathrm{b}}$ \\
& $21,79^{\mathrm{b}}$ & 4,23 & $( \pm 0,19)$ & $( \pm 0,07)$ & $( \pm 0,07)$ & $( \pm 1,70)$ & $( \pm 0,20)$ \\
\hline 100 & $(0,75)$ & & $\begin{array}{c}13,00^{\mathrm{a}} \\
( \pm 0,80)\end{array}$ & $\begin{array}{c}92,39^{\mathrm{a}} \\
( \pm 0,08)\end{array}$ & $\begin{array}{r}7,61^{\mathrm{d}} \\
( \pm 0,08)\end{array}$ & $\begin{array}{c}44,85^{\mathrm{c}} \\
( \pm 0,94)\end{array}$ & $39,51^{\mathrm{b}}$ \\
& $22,87^{\mathrm{b}}$ & 4,01 & $\pm 1,22)$ \\
\hline
\end{tabular}

MS: materia seca; PC: proteína cruda; MO: materia orgánica; CE: cenizas; FDN: fibra detergente neutro; FDA: fibra detergente ácido. Valor entre paréntesis: desviación estandar / MS: dry matter; PC: crude protein; MO: organic matter; CE: ash; FDN: neutral detergent fiber; FDA: acid detergent fiber. Value in parenthesis: standard deviation.

*Medias en la misma columna con letras distintas difieren estadísticamente $(\mathrm{p}<0,05) /$ Means in the same column with different letters differ statistically $(\mathrm{p}<0.05)$.

Cuadro 3. Contenido de taninos condensados, glucósidos cianogénicos, saponinas y cafeína (\%) en ensilajes elaborados con diferentes porcentajes de pulpa de café (Coffea arabica L.) en sustitución de Pennisetum purpureum var OM-22 en Villaflores, Chiapas, México durante 2015.

Table 3. Content of condensable tannins, cyanogenic glycosides, saponins and caffeine $(\%)$ of silage made with different percentages of pulp coffee (Coffea arabica L.) in place of Pennisetum purpureum var OM-22 in Villaflores, Chiapas, México during 2015.

\begin{tabular}{ccccc}
\hline $\begin{array}{c}\text { Tratamientos } \\
\text { (\%pulpa) }\end{array}$ & TC & GC & SA & Cafeína \\
\hline 0 & 0,00 & Neg & Neg & --- \\
25 & 0,00 & Neg & Neg & $0,23^{\mathrm{d}^{*}}( \pm 0,03)$ \\
50 & 0,00 & Neg & Neg & $0,32^{\mathrm{c}}( \pm 0,04)$ \\
75 & 0,00 & Neg & Neg & $0,47^{\mathrm{b}}( \pm 0,02)$ \\
100 & $1,68( \pm 0,10)$ & Neg & Neg & $0,58^{\mathrm{a}}( \pm 0,02)$ \\
\hline
\end{tabular}

TC: taninos condensables; GC: glucósidos cianogénicos; SA: saponinas; Neg: negativo. Valor entre paréntesis: desviación estándar / TC: condensable tannins; GC: cyanogenic glycosides; SA: saponins; Neg: negative. Value in parenthesis: standard deviation. *Medias en la misma columna con letras distintas difieren estadísticamente $(\mathrm{p}<0,05) /$ Means in the same column with different letters differ statistically $(\mathrm{p}<0.05)$. a sustancias pépticas totales, azúcares reductores, azúcares no reductores, cafeína, ácido clorogénico y ácido cafeico total son responsables de la toxicidad de la pulpa de café (Bressani, 1978). Este trabajo reportó valores de $1,68 \%$ para aquel ensilaje que contenía solamente pulpa de café $(100 \%)$, sin embargo, para los otros niveles de inclusión de la pulpa $(25,50,75 \%)$ no se detectó. Este comportamiento ha sido descrito por distintos autores (Gómez et al., 1985; Ferrer et al., 1995). Posiblemente, el proceso de fermentación en el silo pudo inactivar biológicamente a los taninos, por lo que se presume que el hecho de tener la pulpa de café en fermentación estimula la actividad de las reacciones enzimáticas que pueden convertirlas en sustancias inocuas (Noriega et al., 2009), o bien, la disminución puede deberse a que el compuesto es soluble al agua y se pierde en los líquidos del drenado (Murillo, 1978).

El valor encontrado para TC en este trabajo difiere a los reportados por otros autores (Yonatan et al., 2011) quienes han encontrado valores cuyo rango fue de 0,34 a $0,50 \%$ para niveles de sustitución de pasto por pulpa de café de 10 hasta $100 \%$, sin embargo, dicha diferencia podría estar asociada al origen de la pulpa de café, ya que en el presente trabajo la pulpa fue obtenida de beneficio húmedo, la cual pierde la 
mayoría de azúcares, compuestos fenólicos y otros compuestos solubles al agua (Ramírez et al., 2004). De aquí que la composición de la pulpa de café ensilada que se produce depende en gran parte del origen y el manejo de esta al ser ensilada.

Respecto a los valores de cafeína, los resultados indicaron que existió un aumento significativo $(\mathrm{p}<0,05)$ de este compuesto conforme la pulpa de café sustituyó al pasto $P$. purpureum, tendencia similar a lo reportado por Yonatan et al. (2011). El valor encontrado para el tratamiento con $100 \%$ de pulpa de café ensilada $(0,58 \%)$ estuvo cercano al rango reportado en microsilos de pulpa de café utilizando distintos aditivos (0,42-0,56\%) (Ramírez et al., 1999), pero fue menor a los valores reportados por Ulloa et al. (2003) (1,30\%) y Munguía (2015) (2,5\%). Sin embargo, estos valores podrían no representar problemas, puesto que, a pesar de que la cafeína se considera un factor limitante en la alimentación animal, algunos trabajos han reportado que su consumo por rumiantes a través de diferentes niveles de pulpa de café ensilada no ha presentado efectos negativos (Souza et al., 2005; Salinas et al., 2015), además de que se tiene evidencia de que los valores de cafeína se reducen significativamente en períodos largos de ensilaje (Porres et al., 1993), producto del proceso de fermentación llevado a cabo (Moreau et al., 2003).

Sobre la conducta ingestiva se obtuvieron los valores más altos $(\mathrm{p}<0,05)$, para velocidad de bocados (VB) y velocidad de consumo (VC), para los ensilajes con 25 y $50 \%$ de inclusión de pulpa de café; sin embargo, el tamaño de bocado (TB) no difirió entre tratamientos $(\mathrm{p}>0,05)$ (Cuadro 4).

En función del índice de preferencia calculado, los tratamientos que más prefirieron los animales, fueron los que incluyeron 25 y $50 \%$ de pulpa de café en el ensilaje $(p<0,05)$, con valores de 0,40 y 0,35 , respectivamente. Los valores para los tratamientos 0,75 y $100 \%$ fueron similares entre sí con un valor común de 0,07 .

Con respecto a lo encontrado en la evaluación de conducta ingestiva, los resultados indican que la velocidad con la que el ensilado fue consumido (VB y VC), explica diferencias en la conducta animal sobre el uso de este tipo de suplemento, es decir, los animales consumieron de manera más rápida los ensilados fabricados con 25 y $50 \%$ de pulpa, pero disminuyó su VB y VC conforme el porcentaje de pulpa de café aumentó en el ensilaje, resultado posiblemente asociado al sabor conferido por la pulpa,
Cuadro 4. Conducta ingestiva en bovinos suplementados con microsilos elaborados con distintas proporciones de pulpa de café (Coffea arabica L.) en sustitución de Pennisetum purpureum var OM-22 en Villaflores, Chiapas, México durante 2015.

Table 4. Ingestive behavior of cattle supplemented with microsilos made with different percentages of pulp coffee (Coffea arabica L.) in place of Pennisetum purpureum var OM-22 in Villaflores, Chiapas, México during 2015.

\begin{tabular}{lccc}
\hline $\begin{array}{l}\text { Tratamientos } \\
\text { (\% pulpa) }\end{array}$ & \multicolumn{3}{c}{ Variables } \\
\cline { 2 - 4 } & VB & VC & TB \\
\hline 0 & $1,68 \mathrm{~b}^{*}$ & $2,93 \mathrm{bc}$ & $5,81 \mathrm{a}$ \\
25 & $9,25 \mathrm{a}$ & $10,20 \mathrm{a}$ & $1,48 \mathrm{a}$ \\
50 & $6,78 \mathrm{a}$ & $8,81 \mathrm{ab}$ & $1,60 \mathrm{a}$ \\
75 & $0,56 \mathrm{~b}$ & $1,74 \mathrm{c}$ & $4,11 \mathrm{a}$ \\
100 & $0,32 \mathrm{~b}$ & $1,85 \mathrm{c}$ & $5,08 \mathrm{a}$ \\
Promedio & 3,72 & 5,11 & 3,61 \\
EEM & 1,11 & 0,97 & 1,63 \\
\hline
\end{tabular}

VB: velocidad de bocados (bocados/min); VC: velocidad de consumo (g MS/min); TB: tamaño de bocado (g MS/bocado); EEM: error estándar de la media / VB: speed bite (bites/min); VC: consumption rate (g DM/min); TB: size bites (g DM/bite); EEM: standard error of the mean.

* Medias en la misma columna con letras distintas difieren estadísticamente $(\mathrm{p}<0,05) /$ Means in the same column with different letters differ statistically $(\mathrm{p}<0.05)$

aspecto que toma interés en los procesos de adaptación al suplemento por parte del animal; sin embargo, la cantidad de ensilaje ingerido por mordida (TB) fue similar en todos los tratamientos. Lo encontrado podría ser una respuesta a las distintas características físico-químicas del ensilaje, lo que provocó que las velocidades de consumo fueran diferentes, ya que factores, tales como el sabor, olor, textura y composición química afectan la conducta ingestiva del animal (Kaitho, 1997). Estos resultados corroboran la importancia de involucrar el componente animal en las evaluaciones iniciales de cualquier alimento.

Respecto al índice de preferencia, los resultados fueron relevantes para los tratamientos con inclusión de pulpa de café en un rango entre 25 y $50 \%$. Lo anterior podría indicar que estos tratamientos correspondieron a los preferidos por los animales, ya que para su cálculo se involucró el consumo que tuvo el tratamiento entre el consumo total de todos los tratamientos. 


\section{LITERATURA CITADA}

Altmann, J. 1974. Observational study of behavior: sampling methods. Behavior 49:227-265.

AOAC (Association of Official Analytical Chemists). 1990. Official methods of analysis. $15^{\text {th }}$ ed. AOAC, Arlington, VA, USA.

ARD (Agricultural Rural Development). 2004. Visual evaluation of silage quality. Government of Alberta. http://archive.org/stream/silagemanual00maca (accesed 29 nov. 2015).

Arellano, M.A. 2009. Estimación de la capacidad antioxidante de ácidos hidroxicinámicos obtenidos de la pulpa de café. Tesis MSc., Universidad Autónoma Metropolitana, MEX.

Arrázola, G. 2002. Análisis de glucósidos cianogénicos en variedades de almendro. Implicaciones en la mejora genética. Tesis Dr., Universidad de Alicante, ESP.

Ashbell. G., T. Kipnis, M. Titterton, Y. Hen, A. Azrieli, and A.Z. Weinberg. 2001. Examination of a technology for silage in plastic bags. Anim. Feed Sci. Tech. 91:213-22.

Bautista, O., J. Pernía, D. Barrueta, y M. Useche. 2005. Pulpa ecológica de café ensilada en la alimentación de alevines del híbrido de cachamay (Colossoma macropomun x Piaractus brachypomus). Rev. Cien. Fac. Cien. Vet. LUZ. 15(1):33-40.

Bressani, R. 1978. Posibles usos de los subproductos de grano de café. En: R. Bressani, y J.C. Braham, editores, Pulpa de café: composición, la tecnología y la utilización. Instituto de Nutrición de Centroamérica y Panamá (INCAP), PAN. p. 17-24.

Cárdenas, M.J.V., C.A. Sandoval, y F.J. Solorio. 2003. Composición química de ensilajes mixtos de gramíneas y especies arbóreas de Yucatán, México. Tec. Pecu. Mex. 41:283-294.

Clifford, M.N. 1985. Chlorogenics acids. In: R.J. Clarke, and R. Maurae, editors, Coffee. Vol. 1. Chemistry. Elsevier Applied Science Publisher, London, GBR. p. 153-202.

Corro, G., L. Paniagua, U. Pal, F. Banuelos, and M. Rosas. 2013. Generation of biogas from coffee-pulp and cowdung codigestion: Infrared studies of postcombustion emissions. Energy Convers. Manag. 74:471-481.

Ferrer J., G. Páez, M. Chirino, y Z. Mármol. 1995. Ensilaje de la pulpa de café. Rev Fac. Agron. LUZ 12:417-428.

García, E. 1989. Modificación al sistema de clasificación climática de Köppen. Universidad Nacional Autónoma de México, MEX.

Gómez R., G. Bendaña, J. González, E. Braham, y R. Bressani. 1985. Relación entre los niveles de inclusión de la pulpa de café y contenido proteínico en raciones para animales monogástricos. Arch. Lat. Nutr. 35:422-437.

González, N. 1990. Alimentación animal. América C.A., Ciudad de México, MEX.

Kaitho, R.J. 1997. Nutritive value of browses as protein supplement (s) to poor quality roughages. Ph.D. Tesis, Wageningen Agricultural University, Wageningen, HOL.

Kassa, H., H. Suliman and T. Workayew. 2011. Evaluation of composting process and quality of compost from coffee by products (coffee husk and pulp). Ethiop. J. Env. Stud. Manag. 8:616-627.

Menezes, E., J. Do Carmo, J.G. Alves, A. Menezes, I.C. Guimaraes, F. Queiroz, and C.J. Pimienta. 2014. Optimization of alkaline pretreatment of coffee pulp for production of bioethanol. Biotechnol. Progr. 30:451-462.

Molina, M., R. Lechuga, y R. Bressani. 1990. Valor nutritivo de la pulpa de café sometida a fermentación sólida usando Aspergillus niger en pollos y cerdos. Agron. Mesoam. 1:79-82.

Moreau, Y., J.L. Arredondo, I. Perroud, and S. Roussos. 2003. Dietary utilization of protein and energy from fresh and ensiled coffee pulp by the Nile Tilapia Oreochromis niloticus. Brazilian Arch. Biol. Technol. 46:223-231.

Morris, B., 1973. The chemical analysis of foods and foods products, $3^{\text {rd }}$ ed. Robert Kriger Publishing Co., NY, USA.

Munguía, G. 2015. Comportamiento, producción y características de la canal en ovinos alimentados con pulpa de café. Tesis MSc., Colegio de Posgraduados, Montecillo, MEX.

Murillo, B. 1978. Ensilaje de pulpa de café. En: R. Bressani, y J.C. Braham, editores, Pulpa de café: composición, la tecnología y la utilización, publicación. Instituto de Nutrición de Centroamerica y Panamá (INCAP), PAN.

Noriega, S.A., A.R. Silva, y M. García. 2008. Revisión. Utilización de la pulpa de café en la alimentación animal. Zootec.Trop. 26:411-419.

Noriega, S.A., A.R. Silva, y M. García. 2009. Composición química de la pulpa de café a diferentes tiempos de ensilaje para su uso potencial en la alimentación animal. Zootec. Trop. 27:135-141.

Nurfeta, A. 2010. Feed intake, digestibility, nitrogen utilization and body weight change of sheep consuming wheat straw supplemented with local agricultural and agro-industrial by-products. Trop. Anim. Health Prod. 42:815-824.

Ojeda, G.F., G.O. Cáceres, y M. Esperance. 1991. Conservación de forrajes. Editorial Pueblo y Educación, La Habana, CUB. 
Ortega, L., y F. Rivas. 1998. Acondicionamiento aversivo de ovejas para evitar el consumo de planta de naranja Citrus sinensis. Téc. Pecu. Méx. 36:49-58.

Pedraza, B.P., F.J. Estrada, C.A. Martínez, L.I. Estrada, A.A. Rayas, G. Yong, M.M. Figueroa, N.F. Avilés, and O. Castelán. 2012. On-farm evaluation of the effect of coffee pulp suplementation on milk yield and dry matter intake of dairy cows grazing tropical grasses in central Mexico. Trop. Anim. Health Prod. 44:329-336.

Pinto, R., D. Hernández, F. Guevara, H. Gómez, F. Medina, A. Hernández, J.A. Jiménez, E. Alfonso, P. Mendoza, y B. Ruiz. 2010. Preferencia de ovinos por el ensilaje de Pennisetum purpureum mezclado con arbóreas forrajeras tropicales. Livest. Res. Rural Dev. 22(6). http://www.lrrd.org/lrrd22/6/pint22106.htm

Pinto, R.R., A. Ley, F. Guevara, A. Hernández, J. Carmona, y D. Hernández. 2016. Incorporación de subproductos agropecuarios en la alimentación de rumiantes. Universidad Central Martha Abreu de las Villas, Cayo Santa María, CUB.

Porres, C., D. Alvarez, and J. Calzada. 1993. Caffeine reduction in coffee pulp through silage. Biotech. Adv. 11:519-523.

Preston, T.R., y R.A. Leng. 1990. Ajustando los sistemas de producción pecuaria a los recursos disponibles: Aspectos básicos y aplicados del nuevo enfoque sobre la nutrición en el trópico. Círculo de impresores Ltda., Cali, COL.

Ramírez, M.A., N. Marnet, S.K. Kolli, S. Roussos, S. Guyot, and C. Augur. 2004. Characterization and estimation of proanthocyanidins and other phenolics inn coffee pulp by thiolysis-high-performance liquid chromatography. J. Agric. Food Chem. 52:1344-1349.

Ramírez, M.J.R., R.D. Pernía, E.O. Bautista, M.N. Clifford, y M.R. Adams. 1999. Producción y caracterización de la Pulpa de café ensilada. En: J.R. Martínez, editor, Pulpa de café ensilada: producción, caracterización y utilización en la producción animal. Universidad Nacional Experimental del Táchira, San Cristóbal, VEN. p. 139.

Rathinavelu, R., y G. Graziosi. 2005. Posibles usos alternativos de los residuos y subproductos del café. Organización Internacional del Café, ITA.

SAGARPA (Servicio de Información Agroalimentaria y Pesquera). 2011. Producción de café cereza en Chiapas. SAGARPA, MEX.

Salinas, R.T., M.E. Ortega, M.T. Sánchez, J. Hernández, A. Díaz, J.L. Figueroa, R. Guinzberg, and J.L. Cordero. 2015. Productive performance and oxidative status of sheep fed diets supplemented with coffee pulp. Small Rum. Res. 123:17-21.

Salmones, D., G. Mata, and K.N. Waliszewski. 2005. Comparative culturing of Pleurotus spp. on coffee pulp and wheat straw: Biomass production and substrate biodegradation. Bioresour. Technol. 96:537-544.

SAS. 1994. User's guide. $4^{\text {th }}$ ed. SAS Institute Inc., NC, USA. Solomon, D. 1991 The value of coffee pulp alone and in combination with other feeds in sheep nutrition in Ethiopia. Small Ruminant Res. 5:223-231.

Sosa, R.E., D. Pérez, L. Ortega, y G. Zapata. 2004. Evaluación del potencial forrajero de árboles y arbustos tropicales para la alimentación de ovinos. Téc. Pecu. Méx. 42:129-144.

Souza, A.L., R. García, D.C. Valadares, F. Cipriano, J.M. de Souza, L. da Silva, e K.F. Gobbi. 2005. Casca de café em dieta de vacas em lactacao: consumo, digestibilidade e producao de leite. Rev. Bras. Zoot. 34:2498-2504.

Terrill, T.H., M.A. Rowan, B.G. Douglas, and N.T. Barry. 1992. Determination of extractable and bound condensed tannin concentrations in forage plants, protein concentrate meal and cereal grains. J. Sci. Food Agric. 58:321

Torres, M.T., J. Córdova, S.G. Rodríguez, S. Roussos, C.A. Ramírez, T.E. Favela, and G. Saucedo. 2011. Enzymatic extraction of hidroxycinnamic acids from coffee pulp. Food Tech. Biotechnol. 49:369-373.

Ulloa, J.B., J.A.J. Verneth, S. Amato, and E.A. Huisman. 2003. Biological treatments affect the chemical composition of coffee pulp. Bioresources Technol. 89:267-274.

Van Soest, P.J., J.B. Robertson, and B.A. Lewis. 1991. Methods for dietary fiber, neutral detergent fiber and non-starch polysaccharides in relation to animal nutrition. J. Dairy Sci. 74:3583-3597.

Villalba, D.K., V.A. Holguín, J.A. Acuña, y V.R. Piñeros. 2011. Calidad bromatológica y organoléptica de ensilajes de residuos orgánicos del sistema de producción café-musáceas. Rev. Col. Cienc. Anim. 4(1):47-52.

Yonatan, K., D. Solomon, and T. Taye. 2011. Chemical composition and in-vitro digestibility of coffee pulp ensiled with effective microorganism in Ethiopia. Livest. Res. Rural Dev. 23(7). http://www.lrrd.org/ $\operatorname{lrd} 23 / 7 / \mathrm{kass} 23155 . \mathrm{htm}$ 
